\title{
A Double-Blind Placebo-Controlled Trial of Maca Root as Treatment for Antidepressant-Induced Sexual Dysfunction in Women
}

\author{
Christina M. Dording, ${ }^{1}$ Pamela J. Schettler, ${ }^{2}$ Elizabeth D. Dalton, ${ }^{1}$ Susannah R. Parkin, ${ }^{1}$ \\ Rosemary S. W. Walker, ${ }^{1}$ Kara B. Fehling, ${ }^{1}$ Maurizio Fava, ${ }^{1}$ and David Mischoulon ${ }^{1}$ \\ ${ }^{1}$ Depression Clinical and Research Program, Department of Psychiatry, Massachusetts General Hospital, Boston, MA 02114, USA \\ ${ }^{2}$ Department of Psychiatry and Behavioral Sciences, Emory University, Atlanta, GA 30322, USA
}

Correspondence should be addressed to Christina M. Dording; cdording@partners.org

Received 17 December 2014; Revised 11 March 2015; Accepted 23 March 2015

Academic Editor: Sonia Piacente

Copyright ( $) 2015$ Christina M. Dording et al. This is an open access article distributed under the Creative Commons Attribution License, which permits unrestricted use, distribution, and reproduction in any medium, provided the original work is properly cited.

\begin{abstract}
Objective. We sought to demonstrate that maca root may be an effective treatment for antidepressant-induced sexual dysfunction (AISD) in women. Method. We conducted a 12-week, double-blind, placebo-controlled trial of maca root (3.0 g/day) in 45 female outpatients (mean age of $41.5 \pm 12.5$ years) with SSRI/SNRI-induced sexual dysfunction whose depression remitted. Endpoints were improvement in sexual functioning as per the Arizona Sexual Experience Scale (ASEX) and the Massachusetts General Hospital Sexual Function Questionnaire (MGH-SFQ). Results. 45 of 57 consented females were randomized, and 42 (30 premenopausal and 12 postmenopausal women) were eligible for a modified intent-to-treat analysis based on having had at least one postmedication visit. Remission rates by the end of treatment were higher for the maca than the placebo group, based on attainment of an ASEX total score $\leq 10$ (9.5\% for maca versus $4.8 \%$ for placebo), attaining an MGH-SFQ score $\leq 12$ (30.0\% for maca versus $20.0 \%$ for placebo) and reaching an MGH-SFQ score $\leq 8$ (9.5\% for maca versus 5.0\% for placebo). Higher remission rates for the maca versus placebo group were associated with postmenopausal status. Maca was well tolerated. Conclusion. Maca root may alleviate SSRI-induced sexual dysfunction in postmenopausal women. This trial is registered with NCT00568126.
\end{abstract}

\section{Introduction}

Antidepressant-induced sexual dysfunction (AISD) is a significant complication in the treatment of patients with mood and anxiety disorders, affecting more than half of patients who are taking selective serotonin reuptake inhibitors (SSRIs) or serotonin-norepinephrine reuptake inhibitors (SNRIs) [1], and has been associated with reduced quality of life, reduced self-esteem, and negative effects on mood and relationships [1]. Sexual dysfunction may affect any phase of the sexual response cycle including libido, arousal, and/or orgasm [2]. Because adequate sexual function is an integral aspect of quality of life, many patients whose depression is in remission as a result of taking antidepressant medication may elect to switch or discontinue their treatment, increasing their risk of complications from their underlying psychiatric disorder [3]. AISD is a major reason for medication discontinuation [4].

In the postmenopausal population there are few treatments available for sexual dysfunction regardless of etiology, and none are approved by the FDA for this indication. There is some evidence to support the use of the testosterone patch, though safety concerns restrict its availability in the US [5]. Bupropion has been studied largely in premenopausal women with sexual dysfunction with modest evidence of efficacy $[6,7]$. While a recent study has suggested a benefit from the use of the phosphodiesterase inhibitors in premenopausal women suffering from AISD [8], a recent meta-analysis has demonstrated inconsistent effects of the phosphodiesterase inhibitors in women [9]. Given the limited options for women with AISD, many have turned to complementary and natural 
treatments in the hope of improving sexual functioning [10]. One such natural agent is maca root (Lepidium meyenii).

Maca is a hardy perennial plant cultivated in the Andean Mountains that is traditionally used for both nutritional and fertility-enhancing purposes [11]. Animal studies have demonstrated improvement in sexual behaviors such as increased copulatory attempts due to maca [12-18] and anecdotal evidence certainly supports the use of maca for the treatment of sexual dysfunction in humans [11]. However, few controlled clinical trials in humans have been conducted and those few have generally studied only males. In humans, maca has been shown to increase sperm count and sperm motility [19], as well as increasing sexual desire [20-23]. However the mechanism by which maca exerts its purported aphrodisiac and fertility-enhancing properties is unclear.

In one of the few published studies of maca in women, early postmenopausal women treated with maca were more likely than those who received placebo to show significant decreases in follicle stimulating hormone (FSH) and significant increases in luteinizing hormone $(\mathrm{LH})$ production [24]. In addition, several studies do at least suggest that maca improves menopausal symptoms [20, 24]. For the postmenopausal female population, while the paucity of data precludes any reasonable inference of effectiveness, let alone mechanism of the effect [25], it is quite possible that maca ameliorates postmenopausal symptoms through an androgenic mechanism. Recently, an interesting case report in the British Medical Journal detailed the case of testosterone assay interference by maca, suggesting that maca contains an as of yet unspecified compound with a similar moiety to the human testosterone molecule and that it may be exerting its androgenic effects through actions at the testosterone receptor on target organs without affecting the level of testosterone or gonadotrophins [26]. This may explain why studies in men demonstrate a lack of effect of maca on serum hormone levels [19, 22, 27, 28].

In a prior trial we recently demonstrated that maca treatment may yield improvement in libido in women with AISD [21]. At a dose of 3 grams per day women described greater sexual activity and more enjoyable sexual experiences. In view of the encouraging preliminary findings, we sought to validate the results of our first open-label dose-finding study by conducting a double-blind placebo-controlled trial of maca root in the treatment of AISD in women. An additional aim of the study was to document the safety and tolerability of maca root. Further we intend to explore the hypothesis that the salutary effects of maca on sexual dysfunction derive from an androgenic response.

\section{Materials and Methods}

2.1. Subjects. This study screened 57 and included 45 remitted depressed female outpatients aged 18-65 (mean age 41.5 years \pm 12.5 ) who were currently suffering from AISD. Patients were required to be in remission from any depressive or anxiety disorder with a score of 9 or less on the 17-item Hamilton Rating Scale for Depression (HAM-D-17) [29] and a score of 9 or less on the Hamilton Rating Scale for Anxiety (HAM-A) [30] to indicate remission. To be enrolled in the study, patients had to have been taking an SSRI, venlafaxine, or a tri/hetero cyclic antidepressant for the treatment of depression at a stable dose for at least 4 weeks. Additionally, they had to have been suffering from clinically significant arousal dysfunction or orgasmic dysfunction for at least four weeks, and the dysfunction had to have emerged subsequent to the use of the currently prescribed antidepressant (determined by selfreport). Lastly, all patients must have been partaking in some form of regular sexual activity (i.e., masturbation, oral sex, intercourse) at least twice monthly prior to antidepressant use and must have been willing to continue sexual activity at least once weekly for the duration of the study.

Patients were excluded if they had been diagnosed with a sexual disorder in the past, were currently receiving another medical or therapeutic form of treatment for their sexual dysfunction, were experiencing sexual dysfunction due to a general underlying medical condition, had experienced recent major relationship changes or turmoil unrelated to the sexual dysfunction, or had any other general health problems or social situations that might have influenced sexual dysfunction or its treatment.

The patients were recruited from December of 2007 through June of 2010 through the Depression Clinical and Research Program in Boston, MA. The Institutional review Board of Massachusetts General Hospital (MGH) approved this study, and all patients gave their written informed consent before study procedures began.

After a diagnostic screening and baseline visit, eligible patients were randomized in a double-blind manner to maca root $1500 \mathrm{mg}$ bid or placebo bid, for 12 weeks. All patients were assessed biweekly using the Massachusetts General Hospital-Sexual Functioning Questionnaire (MGHSFQ) [31] and the Arizona Sexual Experience Scale (ASEX) [32], both five-item rating scales that evaluate the various areas of sexual function (i.e., arousal, orgasm, and satisfaction). Patients were also assessed for degree and improvement of AISD by the Clinical Global Impression-Severity and Clinical Global Impression-Improvement scales (CGI-S and CGI-I) [33], respectively. To monitor depressive, anxious, and other clinical symptoms, we used the 28-item HAM$\mathrm{D}$, the 14-item HAM-A, and the Kellner's Symptoms Questionnaire (SQ) [34]. Additionally, side effects, adverse events, and concomitant medications were recorded by the treating psychiatrist at every visit. A nonblind physician had access to the study randomization list in order to provide appropriate follow-up care to patients who dropped out of the study. At the end of the 12 weeks of the study, patients were offered three months of follow-up care.

Blood samples were obtained from all study participants and assayed for estradiol, progesterone, prolactin, and testosterone, in the MGH Clinical Laboratory.

The maca product we used, including the commercial preparation as well as a sample of the source plant obtained from the Peruvian manufacturer, was analyzed and vouchered by botanists at the Harvard University Herbaria and the Massachusetts College of Pharmacy and Health Sciences (MCPHS). Macamide content was determined by liquid chromatography-tandem mass spectrometry (LCMS) using standard macamides. Our sample consisted of 
N-benzyl-palmitamide $(42.2 \mu \mathrm{g} / \mathrm{g}$ of plant material), N-benzyl-stearamide $(10.6 \mu \mathrm{g} / \mathrm{g}), \mathrm{N}$-benzyl-oleamide $(16.4 \mu \mathrm{g} / \mathrm{g})$, $\mathrm{N}$-(methoxy-benzyl)-oleamide $(2.4 \mu \mathrm{g} / \mathrm{g})$, N-benzyl-linoleamide $(49.8 \mu \mathrm{g} / \mathrm{g}), \mathrm{N}$-(methoxy-benzyl)-linoleamide $(7.3 \mu \mathrm{g} /$ g), N-benzyl-linoleniamide $(53.5 \mu \mathrm{g} / \mathrm{g})$, N-(methoxy-benzyl)-linoleniamide $(4.1 \mu \mathrm{g} / \mathrm{g})$, and $\mathrm{N}$-(methoxy)-benzylpalmitamide $(4.4 \mu \mathrm{g} / \mathrm{g})$. The macamide concentrations were consistent with those in previously analyzed maca samples.

2.2. Statistical Analyses. Analyses were conducted on the basis of a modified intent-to-treat (ITT) sample and consisted of 42 women who completed at least one clinical assessment after a minimum of seven days of treatment. This included 21 women (14 premenopausal and 7 postmenopausal) randomized to treatment with maca and 21 women (16 premenopausal and 5 postmenopausal) randomized to placebo. Analyses were performed for each aggregate treatment arm; subgroup analyses based upon menopausal status were also performed. Significance of change in total ASEX and MGHSFQ scores between baseline and final assessment were assessed by the paired $t$-test.

Treatment groups were also compared based on the percentage reaching either of two remission thresholds per scale: a total score of 12 ("minimally diminished") or less and of 8 ("normal") or less on the MGH-SFQ scale and a total score of 10 ("very strong/very easily/very satisfying") or less and of 8 or less on the ASEX.

Remission rates for maca versus placebo were compared by means of relative risk (if the number meeting remission in the placebo group was greater than zero) and by odds ratio (with 95\% confidence interval). These analyses were repeated for each of the four individual items of the MGHSFQ assessing interest, arousal, orgasm, and satisfaction. For the item analyses, groups were compared on the number and percentage reaching a final status of "minimally diminished" or better relative to their "normal" functioning (self-rating of $1,2$, or 3$)$.

The paired samples $t$-test was used to assess changes in serum testosterone from baseline to endpoint for each treatment arm. Differences between the two treatment arms and between pre- and postmenopausal women were compared using the independent samples $t$-test.

\section{Results}

Mean change in total ASEX and MGH-SFQ (-4.03 for maca and -3.06 for placebo on the MGH-SFQ and -3.84 for maca and -2.11 for placebo on the ASEX) scores was not significantly different for the maca versus placebo groups, either overall or within premenopausal or postmenopausal subgroups. Remission rates were higher for the maca group than the placebo group for attainment of an ASEX total score of $\leq 10$ ( $9.5 \%$ for maca versus $4.8 \%$ for placebo), attaining an MGH-SFQ score of $\leq 12$ (30.0\% for maca versus $20.0 \%$ for placebo) and reaching an MGH-SFQ score of $\leq 8(9.5 \%$ for maca versus $5.0 \%$ for placebo). No women reached a threshold of 8 or less on their total ASEX score. Results are summarized on Table 1.
When adjusted for menopausal status, the higher remission rates for the overall maca versus placebo group were attributable to the postmenopausal women. Among the very small group of postmenopausal women, remission rates by all three total score criteria were notably higher for maca than for placebo: $14.3 \%$ versus $0.0 \%$ for ASEX $\leq$ 10 (Maca/Placebo Odds Ratio $(95 \%$ CI) $=5.553(0.104-$ 295.862)); $57.1 \%$ versus $20.0 \%$ for $\mathrm{MGH} \leq 12$ (Maca/Placebo Odds Ratio (95\% CI) $=5.333$ (0.375-75.779)); and 14.3\% versus $0.0 \%$ for $\mathrm{MGH} \leq 8$ (Maca/Placebo Odds Ratio (95\% CI $=5.553$ (0.104-295.862)). Among premenopausal women, by contrast, remission rates by two measures (ASEX $\leq 10$ and $\mathrm{MGH}-\mathrm{SFQ} \leq 8$ ) were similar for maca and placebo; on the third measure $(\mathrm{MGH}-\mathrm{SFQ} \leq 12)$ the remission rate was considerably lower for maca than for placebo. Results are summarized on Tables 1 and 2. Analysis of specific areas of sexual function showed that, among postmenopausal women, maca improved orgasm (MGH-SFQ item 3), while there was no difference between premenopausal women on maca versus placebo for this item. Among premenopausal (but not postmenopausal women), maca improved arousal (MGH-SFQ item 2). Results are summarized in Table 2.

Advancing age correlated significantly with improvement in sexual functioning as measured by the ASEX in the maca but not in the placebo group $(R=0.626, R$ squared $=0.392$, and $P=0.005$ ).

Change in testosterone level from baseline to endpoint did not correlate significantly with improvements in sexual functioning in the placebo group but did correlate significantly in the maca group as measured by the ASEX $(R=0.834, R$ squared $=0.95$, and $P=0.042)$. Endpoint testosterone levels correlated with improvements in sexual functioning in the maca group alone as measured by the $\operatorname{ASEX}(R=0.720, R$ squared $=0.518$, and $P=0.008)$. The MGH-SFQ trended towards significance with a $P=0.057$.

Exploratory analyses of the hormones were performed, including estradiol, progesterone, and prolactin $(P>0.05$ for all comparisons).

3.1. Tolerability of Maca. The maca treatment was well tolerated overall. Three subjects discontinued their participation in the study due to adverse events, such as flu-like symptoms and vomiting. In all cases, it was difficult to attribute these symptoms to maca.

\section{Discussion}

This is, to our knowledge, the first double-blind placebocontrolled study to examine the efficacy and safety of maca as a treatment for antidepressant-induced sexual dysfunction in women. It expands on the findings of our first openlabel study (in which $85 \%$ of the participants were women) that demonstrated notable mean improvement in sexual function, with the most robust and statistically significant improvement in those subjects receiving 3.0 rather than 1.5 grams of maca per day. In that study, maca also resulted in a significant improvement in libido in the ITT sample, particularly in the high dose group. 


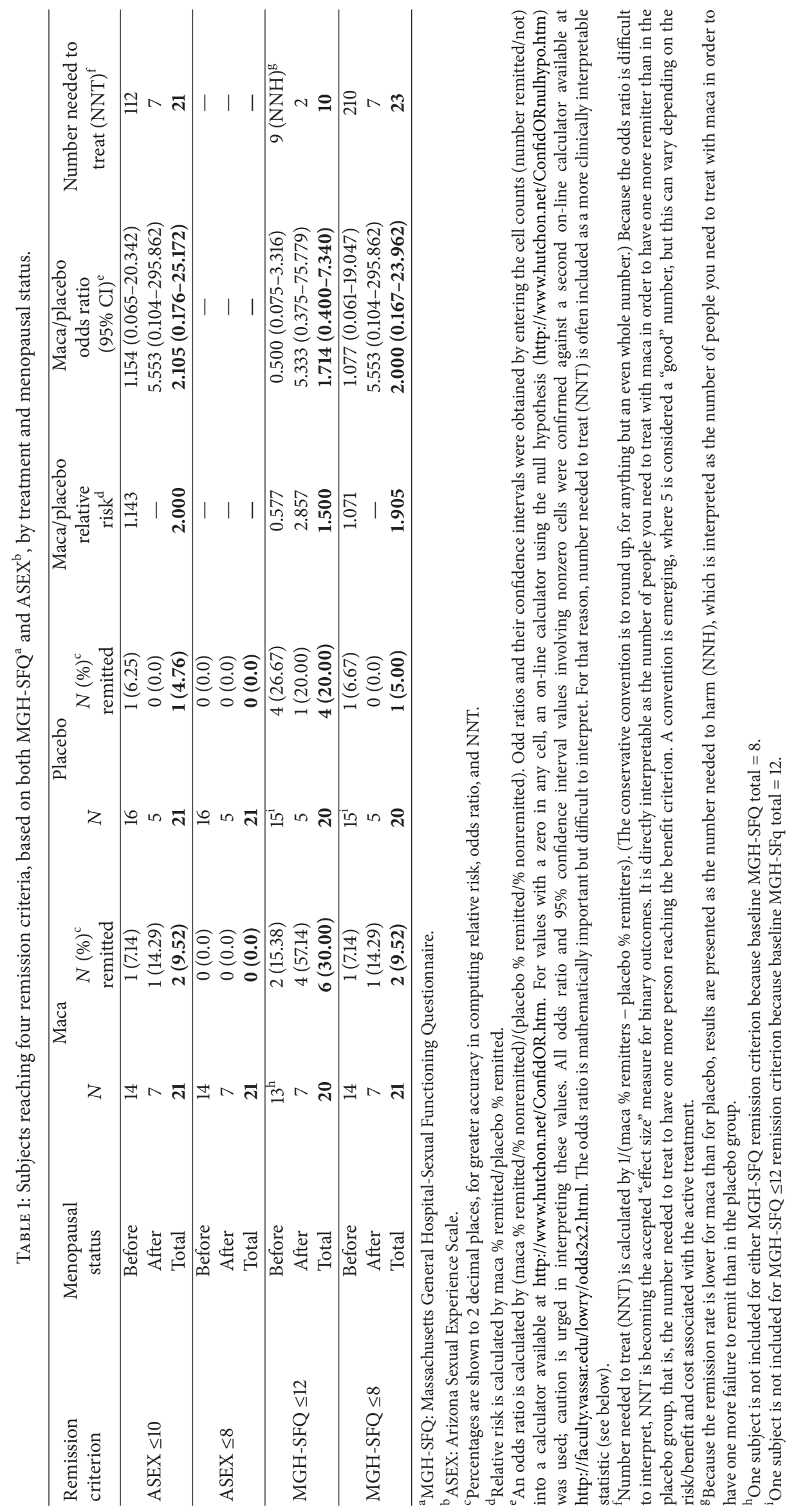




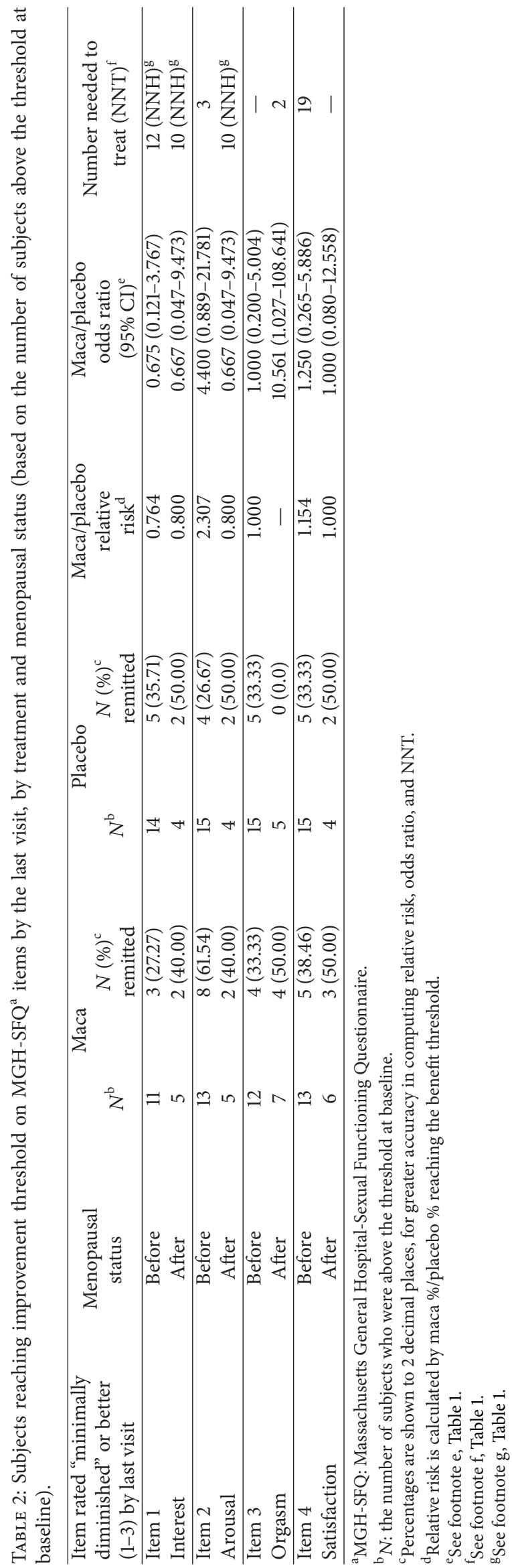


In this double-blind, placebo-controlled, follow-up study, remission rates of sexual dysfunction by the end of treatment were higher for the maca than the placebo group. This difference seemed to be driven by a cohort of postmenopausal women, though we believe that this difference may be more a function of advanced age in the menopausal group rather than by menopausal status itself, as we also found a correlation of improvement in the ASEX sexual dysfunction scores with advancing age and no correlation with estrogen levels that decline precipitously in the postmenopausal period. Testosterone levels are known to gradually decrease with age in general, declining after age 25 and continuing to do so through menopause [35]. We found a significant correlation between change in testosterone and testosterone level at endpoint with improvement in the ASEX in the maca group alone. Of note is the finding from a previous study that postmenopausal women treated with maca were more likely than those who received placebo to show significant decreases in FSH and significant increases in $\mathrm{LH}$ production [24]. We postulate that these changes (in $\mathrm{LH}$ and FSH) via a negative feedback loop result in an increased production of androgens that could explain the improvement in sexual functioning.

Our findings on the relationship between maca and testosterone are interesting and may shed light on the mechanisms of maca. Change in testosterone level from baseline to endpoint and endpoint testosterone levels correlated with improvement in sexual function in the maca group alone. These results seem to be inconsistent with previous findings in men that failed to determine a direct androgenic effect of maca. Clearly however these findings are not generalizable to women; in women it is quite possible that maca may have an indirect androgenic effect via a negative feedback loop involving $\mathrm{LH} / \mathrm{FSH}$. It is clearly difficult to interpret the testosterone findings given the recent suggestion that maca may actually interfere with the testosterone assay and exert its effect through actions at the testosterone receptor on target organs without affecting the level of testosterone or gonadotrophins.

Our study is limited by a number of factors. The sample size was relatively small. Additionally, we relied on patient self-report in the domain of orgasm, which is subjective and may be subject to bias and inaccuracy. Until recently, available methods for measuring the sex steroids have been dependent on antibody based assays that employ a range of different detection systems including the use of isotopes or chemical signaling molecules that produce chemiluminescence. These assays have become increasingly more sensitive for the measurement of testosterone but are still incapable of providing the proper low-end sensitivity for analyzing testosterone in females' blood specimens. Recent advances in the use of ultrasensitive methods such as mass spectrometry coupled to either gas or liquid chromatography have improved the technology for measuring testosterone and other low concentration sex steroids to the degree that mass spectrometry based methods are now capable of measuring testosterone in normal women and in women with extremely low levels of testosterone [35], and running a more sensitive assay for testosterone in women may have elucidated the mechanism of action in this cohort.
Finally, while maca was well tolerated and safe, it bears mentioning that, due to budgetary restrictions, we did not obtain liver function tests (LFTs) for study participants. Many popular herbal extracts may induce elevation in LFTs and, in some extreme cases, mild liver damage. This could be a concern if higher doses are used. Future investigations of maca will include LFTs before and after treatment.

\section{Conclusions}

The current study provides evidence that there may be factors such as androgen levels that mediate the efficacy of maca treatment. Further research, informed by the results of the current study and specifically designed to focus on endocrinologic measures, is required in order to fully examine maca root as a treatment for women with AISD. We are currently developing a larger follow-up study to examine the efficacy of maca root in women with AISD. The follow-up study we are developing will include a more sensitive assay for the measurement of testosterone in women.

In summary, maca root may alleviate AISD as women age, particularly in the domain of orgasm. A larger study in this population using higher doses of maca is in preparation.

\section{Disclosure}

The NCCAM had no further role in study design; in the collection, analysis, and interpretation of data; in the writing of the report; and in the decision to submit the paper for publication.

\section{Disclaimer}

The paper's contents are solely the responsibility of the authors and do not necessarily represent the official views of the National Center for Complementary and Alternative Medicine, National Institutes of Health.

\section{Conflict of Interests}

Christina M. Dording, MD, has received research support from Abbot Laboratories; Alkermes, Inc; Aspect Medical Systems; AstraZeneca; BioResearch; BrainCells Inc.; BristolMyers Squibb; CeNeRx BioPharma; Cephalon; Clinical Trials Solutions, LLC; Clintara, LLC; Covance; Covidien; Eli Lilly and Company; ElMindA, Ltd.; EnVivo Pharmaceuticals, Inc.; Euthymics Bioscience, Inc.; Forest Pharmaceuticals, Inc.; Ganeden Biotech, Inc.; GlaxoSmithKline; Icon Clinical Research; i3 Innovus/Ingenix; Johnson \& Johnson Pharmaceutical Research \& Development; Lichtwer Pharma GmbH; Lorex Pharmaceuticals; National Alliance for Research on Schizophrenia \& Depression (NARSAD); National Center for Complementary and Alternative Medicine (NCCAM); National Institute of Drug Abuse (NIDA); National Institute of Mental Health (NIMH); Novartis AG; Organon Pharmaceuticals; PamLab, LLC; Pfizer Inc.; Pharmavite LLC; Photothera; Roche Pharmaceuticals; RCT Logic, LLC; Sanofi-Aventis US LLC; Shire; Solvay Pharmaceuticals, Inc.; 
Synthelabo; and Wyeth-Ayerst Laboratories. She has served as an advisor/consultant to Takeda. She has received honoraria for speaking and publishing with the American Psychiatric Association, GlaxoSmithKline, MGH Psychiatry Academy, Wyeth-Ayerst Laboratories. Maurizio Fava, MD, has received research support from Abbot Laboratories; Alkermes, Inc.; American Cyanamid; Aspect Medical Systems; AstraZeneca; Avanir Pharmaceuticals; BioResearch; BrainCells Inc.; Bristol-Myers Squibb; CeNeRx BioPharma; Cephalon; Clintara, LLC; Covance; Covidien; Eli Lilly and Company; EnVivo Pharmaceuticals, Inc.; Euthymics Bioscience, Inc.; Forest Pharmaceuticals, Inc.; Ganeden Biotech, Inc.; GlaxoSmithKline; Harvard Clinical Research Institute; Hoffman-LaRoche; Icon Clinical Research; i3 Innovus/Ingenix; Janssen R\&D, LLC; Jed Foundation; Johnson \& Johnson Pharmaceutical Research \& Development; Lichtwer Pharma GmbH; Lorex Pharmaceuticals; Lundbeck Inc.; MedAvante; Methylation Sciences Inc.; National Alliance for Research on Schizophrenia \& Depression (NARSAD); National Center for Complementary and Alternative Medicine (NCCAM); National Institute of Drug Abuse (NIDA); National Institute of Mental Health (NIMH); Neuralstem, Inc.; Novartis AG; Organon Pharmaceuticals; PamLab, LLC; Pfizer Inc.; Pharmacia-Upjohn; Pharmaceutical Research Associates, Inc.; Pharmavite LLC; PharmoRx Therapeutics; Photothera; Reckitt Benckiser; Roche Pharmaceuticals; RCT Logic, LLC (formerly Clinical Trials Solutions, LLC); Sanofi-Aventis US LLC; Shire; Solvay Pharmaceuticals, Inc.; Stanley Medical Research Institute (SMRI); Synthelabo; and Wyeth-Ayerst Laboratories. He has served as an advisor or consultant for Abbott Laboratories; Affectis Pharmaceuticals AG; Alkermes, Inc.; Amarin Pharma Inc.; Aspect Medical Systems; AstraZeneca; Auspex Pharmaceuticals; Bayer AG; Best Practice Project Management, Inc.; BioMarin Pharmaceuticals, Inc.; Biovail Corporation; BrainCells Inc.; BristolMyers Squibb; CeNeRx BioPharma; Cephalon, Inc.; Cerecor; CNS Response, Inc.; Compellis Pharmaceuticals; Cypress Pharmaceutical, Inc.; DiagnoSearch Life Sciences (P) Ltd.; Dinippon Sumitomo Pharma Co. Inc.; Dov Pharmaceuticals, Inc.; Edgemont Pharmaceuticals, Inc.; Eisai Inc.; Eli Lilly and Company; EnVivo Pharmaceuticals, Inc.; ePharmaSolutions; EPIX Pharmaceuticals, Inc.; Euthymics Bioscience, Inc.; Fabre-Kramer Pharmaceuticals, Inc.; Forest Pharmaceuticals, Inc.; GenOmind, LLC; GlaxoSmithKline; Grunenthal $\mathrm{GmbH}$; i3 Innovus/Ingenis; Janssen Pharmaceutica; Jazz Pharmaceuticals, Inc.; Johnson \& Johnson Pharmaceutical Research \& Development, LLC; Knoll Pharmaceuticals Corp.; Labopharm Inc.; Lorex Pharmaceuticals; Lundbeck Inc.; MedAvante, Inc.; Merck \& Co., Inc.; MSI Methylation Sciences, Inc.; Naurex, Inc.; Neuralstem, Inc.; Neuronetics, Inc.; NextWave Pharmaceuticals; Novartis AG; Nutrition 21; Orexigen Therapeutics, Inc.; Organon Pharmaceuticals; Otsuka Pharmaceuticals; Pamlab, LLC; Pfizer Inc.; PharmaStar; Pharmavite LLC; PharmoRx Therapeutics; Precision Human Biolaboratory; Prexa Pharmaceuticals, Inc.; Puretech Ventures; PsychoGenics; Psylin Neurosciences, Inc.; RCT Logic, LLC (formerly Clinical Trials Solutions, LLC); Rexahn Pharmaceuticals, Inc.; Ridge Diagnostics, Inc.; Roche; Sanofi- Aventis US LLC; Sepracor Inc.; Servier Laboratories;
Schering-Plough Corporation; Solvay Pharmaceuticals, Inc; Somaxon Pharmaceuticals, Inc.; Somerset Pharmaceuticals, Inc.; Sunovion Pharmaceuticals; Supernus Pharmaceuticals, Inc.; Synthelabo; Takeda Pharmaceutical Company Limited; Tal Medical, Inc.; Tetragenex Pharmaceuticals, Inc.; TransForm Pharmaceuticals, Inc.; Transcept Pharmaceuticals, Inc.; and Vanda Pharmaceuticals, Inc. He has received honoraria for speaking and/or publishing with Adamed, Co.; Advanced Meeting Partners; American Psychiatric Association; American Society of Clinical Psychopharmacology; AstraZeneca; Belvoir Media Group; Boehringer Ingelheim GmbH; BristolMyers Squibb; Cephalon, Inc.; CME Institute/Physicians Postgraduate Press, Inc.; Eli Lilly and Company; Forest Pharmaceuticals, Inc.; GlaxoSmithKline; Imedex, LLC; MGH Psychiatry Academy/Primedia; MGH Psychiatry Academy/Reed Elsevier; Novartis AG; Organon Pharmaceuticals; Pfizer Inc.; PharmaStar; United BioSource, Corp.; and Wyeth-Ayerst Laboratories. He has a share in equity holdings at Compellis and PsyBrain, Inc. He has patents for Sequential Parallel Comparison Design (SPCD), which are licensed by $\mathrm{MGH}$ to Pharmaceutical Product Development, LLC (PPD), and patent application for a combination of Ketamine plus Scopolamine in Major Depressive Disorder (MDD). He receives copyright royalties for the MGH Cognitive \& Physical Functioning Questionnaire (CPFQ), Sexual Functioning Inventory (SFI), Antidepressant Treatment Response Questionnaire (ATRQ), Discontinuation-Emergent Signs \& Symptoms (DESS), and SAFER; Lippincott, Williams \& Wilkins; Wolkers Kluwer; and World Scientific Publishing Co. Pte. Ltd. David Mischoulon, MD, has received research support from the Bowman Family Foundation, Bristol-Myers Squibb Co., Cederroth, FisherWallace, Ganeden, Lichtwer Pharma, PharmoRx, and Nordic Naturals. He has received honoraria for consulting, speaking, and writing from Pamlab and Nordic Naturals. He has received royalties from Lippincott Williams \& Wilkins for published book Natural Medications for Psychiatric Disorders: Considering the Alternatives. All other authors declare that they have no relevant conflict of interests.

\section{Authors' Contribution}

Christina M. Dording designed the study, wrote the protocol, managed the literature searches and statistical analyses, and wrote the first draft of the paper; Pamela J. Schettler undertook the statistical analyses; Elizabeth D. Dalton coordinated the conduct of the study; Susannah R. Parkin, Rosemary S. W. Walker, and Kara B. Fehling helped in the preparation of the paper; Maurizio Fava oversaw the conduct of the research; and David Mischoulon consulted on the design of the research and statistical analyses and oversaw the writing of the paper.

\section{Acknowledgment}

Dr. Christina M. Dording was supported by an R21 grant from the National Center of Complementary and Alternative Medicine (NCCAM). 


\section{References}

[1] V. S. L. Williams, H. M. Edin, S. L. Hogue, S. E. Fehnel, and D. S. Baldwin, "Prevalence and impact of antidepressant-associated sexual dysfunction in three European countries: replication in a cross-sectional patient survey," Journal of Psychopharmacology, vol. 24, no. 4, pp. 489-496, 2010.

[2] American Psychiatric Association, Diagnostic and Statistical Manual of Mental Disorders, American Psychiatric Association, Washington, DC, USA, 4th edition, 2000.

[3] A. L. Montejo-González, G. Llorca, J. A. Izquierdo et al., "SSRIinduced sexual dysfunction: fluoxetine, paroxetine, sertraline, and fluvoxamine in a prospective, multicenter, and descriptive clinical study of 344 patients," Journal of Sex and Marital Therapy, vol. 23, no. 3, pp. 176-194, 1997.

[4] P. Cassano and M. Fava, "Tolerability issues during long-term treatment with antidepressants," Annals of Clinical Psychiatry, vol. 16, no. 1, pp. 15-25, 2004.

[5] R. Moynihan, "FDA panel rejects testosterone patch for women on safety grounds," The British Medical Journal, vol. 329, no. 7479, article 1363, 2004.

[6] M. R. Safarinejad, S. Y. Hosseini, M. A. Asgari, F. Dadkhah, and A. Taghva, "A randomized, double-blind, placebo-controlled study of the efficacy and safety of bupropion for treating hypoactive sexual desire disorder in ovulating women," $B J U$ International, vol. 106, no. 6, pp. 832-839, 2010.

[7] R. T. Segraves, A. Clayton, H. Croft, A. Wolf, and J. Warnock, "Bupropion sustained release for the treatment of hypoactive sexual desire disorder in premenopausal women," Journal of Clinical Psychopharmacology, vol. 24, no. 3, pp. 339-342, 2004.

[8] H. G. Nurnberg, P. L. Hensley, A. J. Gelenberg, M. Fava, J. Lauriello, and S. Paine, "Treatment of antidepressant-associated sexual dysfunction with sildenafil: a randomized controlled trial," Journal of the American Medical Association, vol. 289, no. 1, pp. 56-64, 2003.

[9] M. L. Chivers and R. C. Rosen, "Phosphodiesterase Type 5 inhibitors and female sexual response: faulty protocols or paradigms?" The Journal of Sexual Medicine, vol. 7, no. 2, pp. 858-872, 2010.

[10] D. Mischoulon, "Polypharmacy and side effects management with natural psychotropic medications," in Natural Medications for Psychiatric Disorders: Considering the Alternatives, D. Mischoulon and J. Rosenbaum, Eds., pp. 207-215, Lippincott Williams \& Wilkins, Philadelphia, Pa, USA, 2002.

[11] R. C. Chacon, A study of the chemical composition of Lepidium meyenii [Dissertation], Universidad Nacional Mayor de San Marcos, Lima, Peru, 1961.

[12] F. Chung, J. Rubio, C. Gonzales, M. Gasco, and G. F. Gonzales, "Dose-response effects of Lepidium meyenii (Maca) aqueous extract on testicular function and weight of different organs in adult rats," Journal of Ethnopharmacology, vol. 98, no. 1-2, pp. 143-147, 2005.

[13] A. F. G. Cicero, E. Bandieri, and R. Arletti, "Lepidium meyenii Walp. Improves sexual behavior in male rats independently from its action on spontaneous locomotor activity," Journal of Ethnopharmacology, vol. 75, no. 2-3, pp. 225-229, 2001.

[14] A. F. G. Cicero, S. Piacente, A. Plaza, E. Sala, R. Arletti, and C. Pizza, "Hexanic Maca extract improves rat sexual performance more effectively than methanolic and chloroformic Maca extracts," Andrologia, vol. 34, no. 3, pp. 177-179, 2002.
[15] G. F. Gonzales, M. Gasco, A. Córdova, A. Chung, J. Rubio, and L. Villegas, "Effect of Lepidium meyenii (Maca) on spermatogenesis in male rats acutely exposed to high altitude (4340 m)," Journal of Endocrinology, vol. 180, no. 1, pp. 87-95, 2004.

[16] G. F. Gonzales, S. Miranda, J. Nieto et al., "Red Maca (Lepidium meyenii) reduced prostate size in rats," Reproductive Biology and Endocrinology, vol. 3, article 5, 2005.

[17] Y. Zhang, L. Yu, M. Ao, and W. Jin, "Effect of ethanol extract of Lepidium meyenii Walp. on osteoporosis in ovariectomized rat," Journal of Ethnopharmacology, vol. 105, no. 1-2, pp. 274-279, 2006.

[18] B. L. Zheng, K. He, C. H. Kim et al., "Effect of a lipidic extract from Lepidium meyenii on sexual behavior in mice and rats," Urology, vol. 55, no. 4, pp. 598-602, 2000.

[19] G. F. Gonzales, A. Cordova, C. Gonzales, A. Chung, K. Vega, and A. Villena, "Lepidium meyenii (Maca) improved semen parameters in adult men," Asian Journal of Andrology, vol. 3, no. 4, pp. 301-303, 2001.

[20] N. A. Brooks, G. Wilcox, K. Z. Walker, J. F. Ashton, M. B. Cox, and L. Stojanovska, "Beneficial effects of Lepidium meyenii (Maca) on psychological symptoms and measures of sexual dysfunction in postmenopausal women are not related to estrogen or androgen content," Menopause, vol. 15, no. 6, pp. 1157-1162, 2008.

[21] C. M. Dording, L. Fisher, G. Papakostas et al., "A double-blind, randomized, pilot dose-finding study of maca root (L. Meyenii) for the management of SSRI-induced sexual dysfunction," CNS Neuroscience and Therapeutics, vol. 14, no. 3, pp. 182-191, 2008.

[22] G. F. Gonzales, A. Córdova, K. Vega et al., "Effect of Lepidium meyenii (MACA) on sexual desire and its absent relationship with serum testosterone levels in adult healthy men," Andrologia, vol. 34, no. 6, pp. 367-372, 2002.

[23] T. Zenico, A. F. G. Cicero, L. Valmorri, M. Mercuriali, and E. Bercovich, "Subjective effects of Lepidium meyenii (Maca) extract on well-being and sexual performances in patients with mild erectile dysfunction: a randomized, double-blind clinical trial," Andrologia, vol. 41, no. 2, pp. 95-99, 2009.

[24] H. O. Meissner, W. Kapczynski, A. Mscisz, and J. Lutomski, "Use of gelatinized maca (Lepidium peruvianum) in early postmenopausal women-a pilot study," International Journal of Biomedical Science, vol. 1, no. 1, pp. 33-45, 2005.

[25] M. S. Lee, B.-C. Shin, E. J. Yang, H.-J. Lim, and E. Ernst, "Maca (Lepidium meyenii) for treatment of menopausal symptoms: a systematic review," Maturitas, vol. 70, no. 3, pp. 227-233, 2011.

[26] L. Srikugan, A. Sankaralingam, and B. McGowan, "First case report of testosterone assay-interference in a female taking maca (Lepidium meyenii)," BMJ Case Reports, 2011.

[27] P. Bogani, F. Simonini, M. Iriti et al., "Lepidium meyenii (Maca) does not exert direct androgenic activities," Journal of Ethnopharmacology, vol. 104, no. 3, pp. 415-417, 2006.

[28] G. F. Gonzalez, A. Córdova, K. Vega, A. Chung, A. Villena, and C. Góñez, "Effect of Lepidium meyenii (Maca), a root with aphrodisiac and fertility-enhancing propeties, on serum reproductive hormone levels in adult healthy men," Journal of Endocrinology, vol. 176, no. 1, pp. 163-168, 2003.

[29] M. Hamilton, "Development of a rating scale for primary depressive illness," The British Journal of Social and Clinical Psychology, vol. 6, no. 4, pp. 278-296, 1967.

[30] M. Hamilton, "The assessment of anxiety states by rating," The British Journal of Medical Psychology, vol. 32, no. 1, pp. 50-55, 1959. 
[31] L. A. Labbate and S. B. Lare, "Sexual dysfunction in male psychiatric outpatients: validity of the Massachusetts General Hospital sexual functioning questionnaire," Psychotherapy and Psychosomatics, vol. 70, no. 4, pp. 221-225, 2001.

[32] C. A. Mcgahuey, A. J. Gelenberg, C. A. Laukes et al., "The arizona sexual experience scale (ASEX): reliability and validity," Journal of Sex and Marital Therapy, vol. 26, no. 1, pp. 25-40, 2000.

[33] W. Guy, ECDEU Assessment Manual for Psychopharmacology, revised, National Institute of Mental Health, Rockville, Md, USA, 1976.

[34] R. Kellner, "A symptom questionnaire," The Journal of Clinical Psychiatry, vol. 48, no. 7, pp. 268-274, 1987.

[35] L. M. Demers, "Androgen deficiency in women: role of accurate testosterone measurements," Maturitas, vol. 67, no. 1, pp. 39-45, 2010 . 


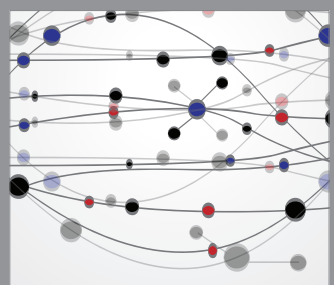

The Scientific World Journal
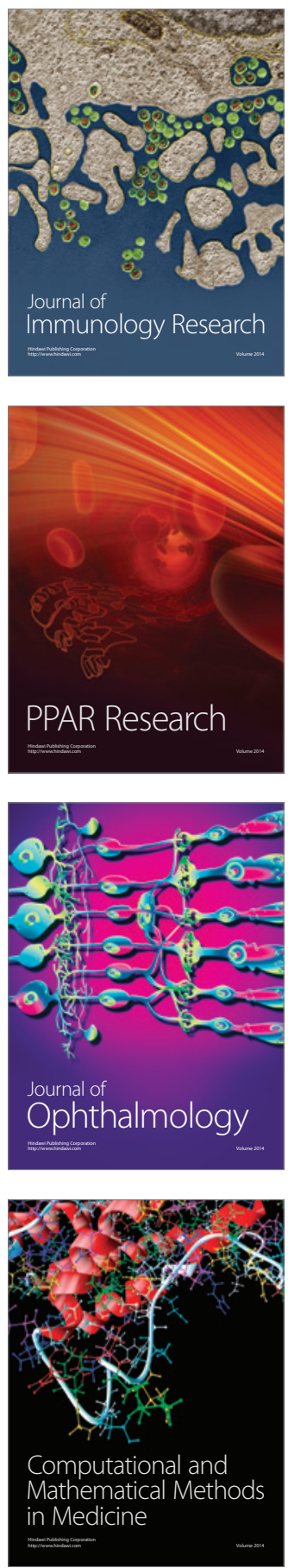

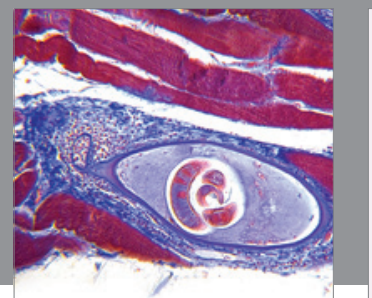

Gastroenterology

Research and Practice
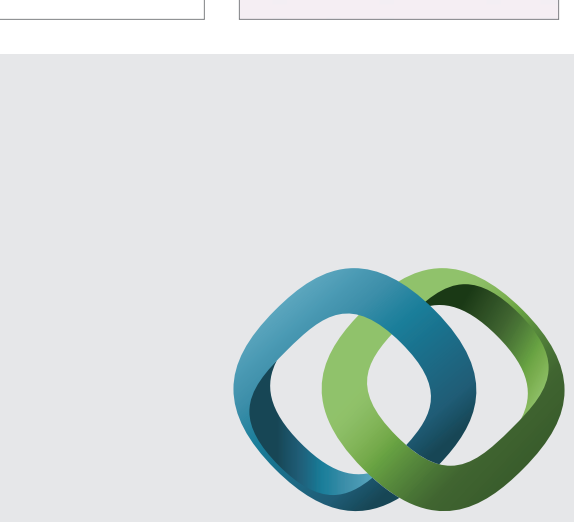

\section{Hindawi}

Submit your manuscripts at

http://www.hindawi.com
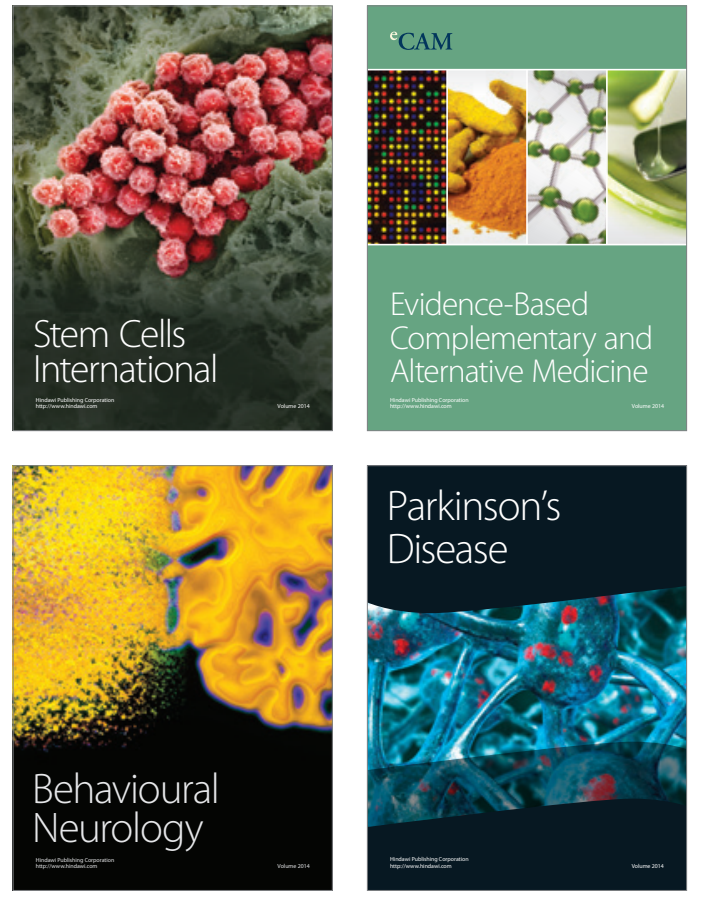
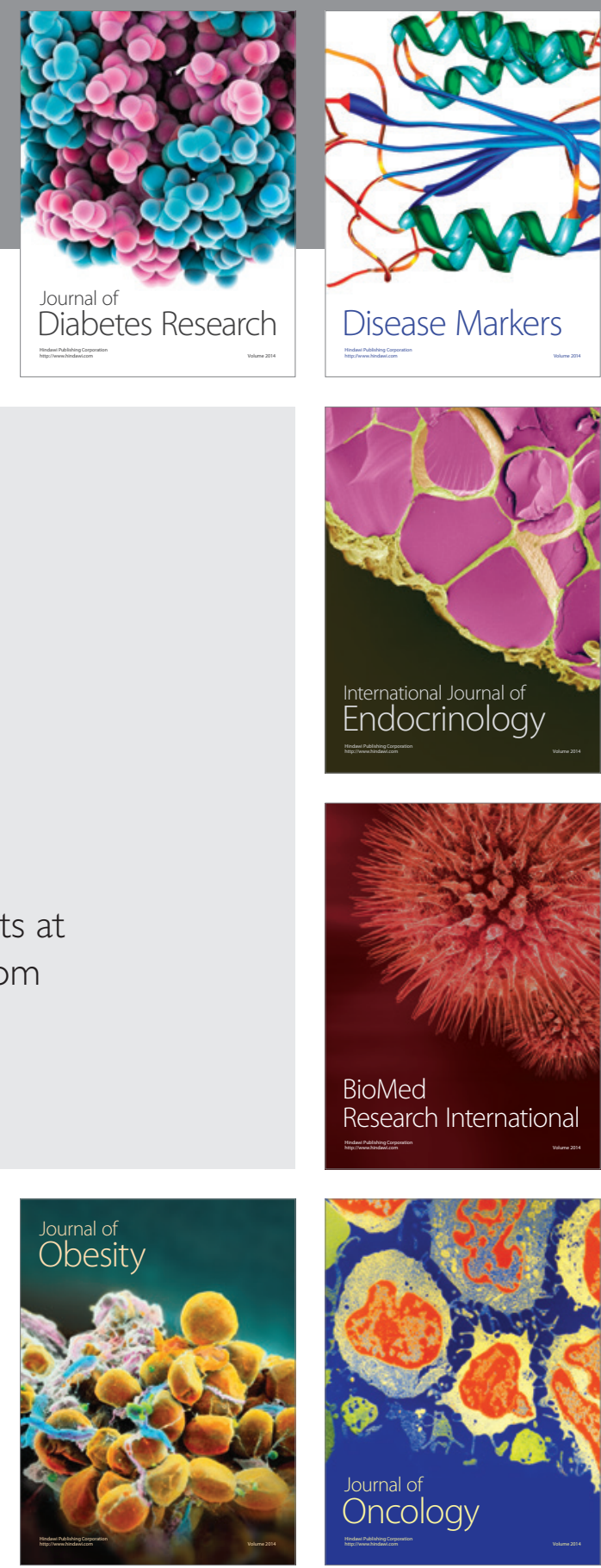

Disease Markers
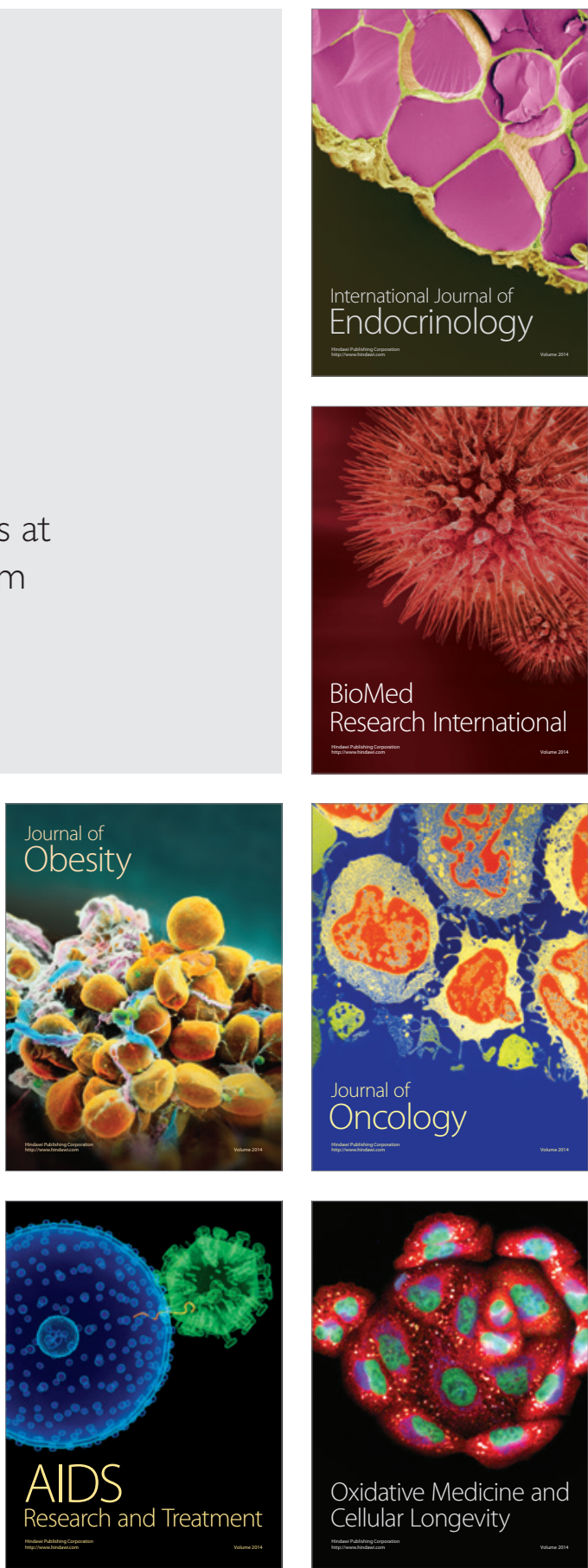